\title{
Web-Based Meaningful Reporting of Patient Harm: Proposal of a Prototype for Creating High Reliability Hospitals
}

\author{
Ranjit Singh1, Raj Sharman², Gayatri Umesh³, K. V. Sankarasubramaniam4, \\ Srikanth Parmeswaran ${ }^{5}$, Arun Nararayan ${ }^{6}$ \\ ${ }^{1}$ Patient Safety Research Center, Department of Family Medicine, State University of New York at Buffalo, \\ Buffalo, USA \\ ${ }^{2}$ UB School of Management, Buffalo, USA \\ ${ }^{3}$ MathWorks, Buffalo, USA \\ ${ }^{4}$ Innovation, Buffalo, USA \\ ${ }^{5}$ Graduate Assistant, Buffalo, USA \\ ${ }^{6}$ Siemens, Buffalo, USA \\ Email: gsingh4@buffalo.edu
}

Received 29 December 2014; accepted 14 January 2015; published 19 January 2015

Copyright (C) 2015 by authors and OALib.

This work is licensed under the Creative Commons Attribution International License (CC BY).

http://creativecommons.org/licenses/by/4.0/

(c) (i)

Open Access

\section{Abstract}

Background: Healthcare settings require a system that detects errors and near misses and learns from them to prevent their reoccurrence. Development of such a system requires engagement of all stakeholders. Retrospective reporting, analyzing and preventing future errors is currently the most prevalent approach to reducing harm to patients. It is important to point out that a recent report regarding reporting by the US Department of Health and Human Services Office of the Inspector General to Congress suggests that "current methods of detection of adverse events are far from adequate and risk misdirection of present efforts to improve safety based quality". Recommended Approach: In an approach to overcome numerous limitations of current reporting methods, the authors propose a web-based user-friendly method that helps engagement of all stakeholders. Whilst invoking "improvement science" with "design science" it applies safety principles. It enables meaningful reporting that applies concepts from organizational models of accidents (based on Reason's Trajectory). The design and use features are described in detail. Conclusion: Pilot tests with this reporting tool have been very encouraging. This trajectory model appears to help reporters to detect and understand that most accidents can be attributed to one or more of three weaknesses: situational, latent, and active.

\section{Keywords}

Barriers to Reporting, Reliability, Safety, System, Trajectory

How to cite this paper: Singh, R., Sharman, R., Umesh, G., Sankarasubramaniam, K.V., Parmeswaran, S. and Nararayan, A. (2015) Web-Based Meaningful Reporting of Patient Harm: Proposal of a Prototype for Creating High Reliability Hospitals. Open Access Library Journal, 2: e1287. http://dx.doi.org/10.4236/oalib.1101287 


\section{Introduction}

Ethical and fiscal imperatives of “First Do No Harm” are well captured by the WHO's declaration that patient safety is a basic human right. Healthcare errors harm one in 10 patients in industrially advanced world [1]. In the US alone about nine million patients are harmed in hospitals [2]. Disability associated with hospitalization occurs in nearly a third of patients older than 70 years. According to the Centers for Disease Control (US CDC), the third leading cause of mortality, after heart disease and cancer, is harm to patients [2]. The US Consumers Union takes the view that preventable adverse events are kept secret from consumers. Minorities of low income seem to be at higher risk. According to the Office of the Inspector General of the US Department of Health and Human Services [3], one in seven Medicare beneficiaries was harmed in 2010. The value of lost lives and disabilities caused by harm in 2006 was estimated to range from 18\% - 45\% of total US healthcare spending [4]. Data on adverse events have been very hard to get because only about $4 \%$ - $5 \%$ of known errors are reported [5]. Opportunist of understanding their causes and design for preventing them are lost [6]. It is not surprising that 13 years after "To Err Is Human” for all practical purposes we have not reduced this huge "iceberg” of harm to patients [7].

What healthcare settings require is a system that detects errors and near misses and learns from them to prevent their reoccurrence. Development of such a system requires engagement of all stakeholders. The Institute of Medicine (IOM), very rightly, asserts that there can be no quality without safety. A 2012 IOM report recommends continuous learning [8]. An August 2013 report to the British Prime Minister by Britain's National Advisory Group on the Safety of Patients in England calls for a promise to learn and a commitment to act to improve safety of patients [9]. These should help fulfill the mission of the US Patient Protection and Affordable Care Act.

Retrospective reporting, analyzing and preventing future errors is currently the most prevalent approach to reducing harm to patients. It is important to point out that a recent report [3] regarding reporting by the US Department of Health and Human Services Office of the Inspector General to Congress suggests that "current methods of detection of adverse events (AEs) are far from adequate and risk misdirection of present efforts to improve safety based quality".

In an approach to overcome numerous limitations of current reporting methods, the authors propose a webbased user-friendly method that helps engagement of all stakeholders. It applies safety principles, whilst invoking "improvement science" with "design science". It enables meaningful reporting that applies concepts from organizational models of accidents (based on Reason's Trajectory) and from high reliability organizations (HRO’s) to healthcare. The main research question is: can the proposed web-based method improve the quality and quantity of error (and close call) reports by all stakeholders at the points of care as well as help develop HRO's?

The first author has already published [8] evidence of successful use of paper version of this trajectory model that has helped reporters to understand that most accidents can be attributed to one or more of three factors/ weaknesses: situational, latent, and active. The error reporters began to understand that the system as a whole produced failures when all individual barriers/weaknesses aligned, permitting "a trajectory of accident opportunity", so that a hazard passed through all of the holes in all of the defenses, leading to a failure/harm. This trajectory has also been used successfully for simulation training. This paper will describe:

- What are the critical factors that are barriers to collection of hospital related errors?

- How do errors propagate in a health care system?

- How can IT systems be designed to make such error reporting easy, highly available, anonymous, and devoid of any attribution of blame?

- How can these errors be classified in a way that provides a deeper understanding of the processes that lead to such errors?

The design and use features are visually described in detail so as to reduce verbosity.

\section{Reporting: Retrospective Approach to Reduction of the Tip of "Iceberg" of Harm}

Retrospective reporting, analyzing and preventing future errors is currently the most prevalent approach to re- 
ducing harm to patients. The overall objective is to reduce the size of the "iceberg", particularly its tip that is revealed (4\% - 5\%) by reporting [10]. This approach has led to development of multitude of reporting methods and development of error taxonomies, at various national and international levels [11]. It is vital that reporting system be perceived by all to be safe (free from blame and shame), easy, and worthwhile [12].

Lately, the profession has realized:

1) the importance of creating a common vision to inculcate team spirit between all stakeholders [13]; this, in turn, demands that we help the teams to overcome current barriers to reporting.

2) the importance of making safety information useful at the point of care to patients and healthcare providers [14]; similarly, the United Kingdom's House of Commons Committee of Public Accounts, in its report calls for a unified and convenient form for reporting that encourages feedback on solutions to specific patient safety incidents. Therefore, in addition to the "macro-system level" data, individual practices/healthcare-sites and organizations need local information that is directly relevant to them and can be used internally to drive safety improvement in each unique site. Such information, reported internally for quality and safety improvement purposes, potentially has more legitimacy in the eyes of local staff and clinicians in any health care setting.

In the US, 2005 Patient Safety and Quality Improvement Act established a federal voluntary database [15]. Federal government is expected to coordinate data collected from Patient Safety Organizations (PSOs) that collect data from hospitals under contract. These PSOs (current total number is about 78) are expected to analyze errors and recommend improvements. The reports are confidential and cannot be used in liability cases. This establishment has six very serious flaws as described below.

1) It is a top-down approach wherein the front line workers and their patient loose the opportunity of understanding of the causes of errors and near misses in their unique setting, and of devising prevention strategies with the resources available to the stakeholders: a bottom-up approach.

2) It lacks transparency to consumer groups.

3) It fails to engage patients. This engagement is the "blockbuster drug of the century" [16].

4) It fails to promote and to enable communication with patients or their families when disclosing adverse events [17] [18]. Disclosure has been shown to decrease lawsuits and average litigation costs [4].

5) It does not help inculcate systems thinking that shuns culture of blame and shame.

6) Most importantly, the reporters face a number of challenges and barriers. This is illustrated in Figure 1.

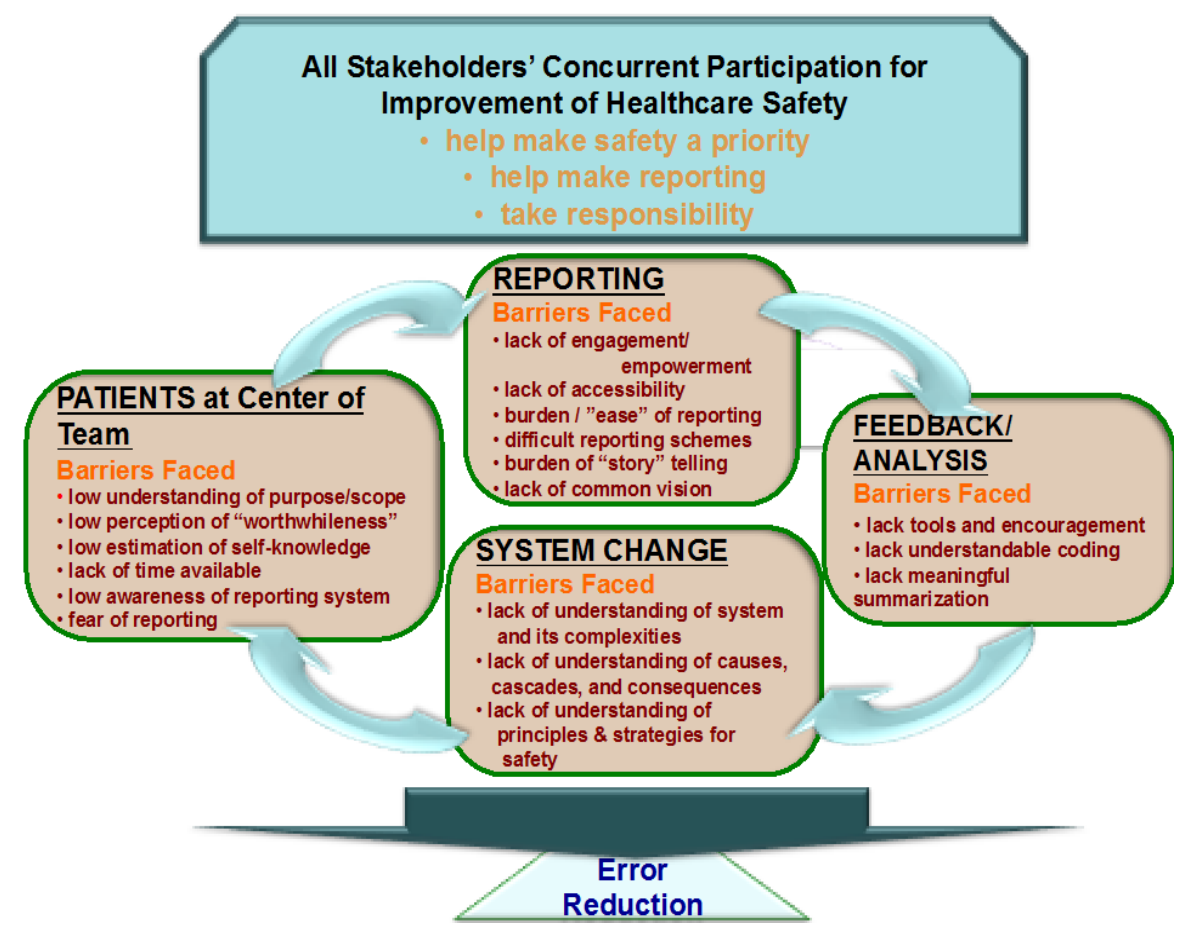

Figure 1. Conceptual model for the error reporting cycle and the barriers faced by potential reporters [9]. 
It is important to point out that a recent report [3] by the US Department of Health and Human Services Office of the Inspector General to Congress on methods for identifying adverse events in hospitals shows concern that incident reporting systems (five different screening methods) are missing the majority of events, and are unreliable. The report suggests that "current methods of detection of AEs are far from adequate and risk misdirection of present efforts to improve safety based quality" [3].

\section{Development of a Concept for Web-Based Meaningful Reporting of Patient Harm}

As stated earlier, what healthcare settings require is a system that detects errors and near misses and learns from them to prevent their reoccurrence. Development of such a system requires, engagement of all stakeholders.

The overarching goal of the authors' interdisciplinary research team is to provide value through qualityimprovement and waste-reduction by applying safety principles and invoking "improvement science" with "design science" portrayed in Figure 2 [19]. In the work presented here, we propose a meaningful reporting methodology that applies concepts from organizational models of accidents [Reason] and from high reliability organizations (HRO's) to healthcare settings.

\subsection{Models of Accident Causation}

Following the spate of disasters occurring in the late 1970s and 1980s, such as Flixborough, Challenger, Three Mile Island, Bhopal, and Chernobyl, James Reason [20] hypothesized that most accidents can be attributed to one or more of three factors/weaknesses: situational, latent, and active. The system as a whole produces failures when all individual barrier weaknesses align, permitting "a trajectory of accident opportunity", so that a hazard passes through all of the holes in all of the defenses, leading to a failure [21]. Singh et al. have successfully used this trajectory model for simulation training in safety.

Figure 3 illustrates Reason's concept of trajectory of errors (accidents).

Figure 4, on the other hand, shows the model that we have adapted to enable its use in reporting and simulation in our past work.

\subsection{Prototype Design}

As a primary requirement the security framework of this prototype complies with Health Insurance Portability

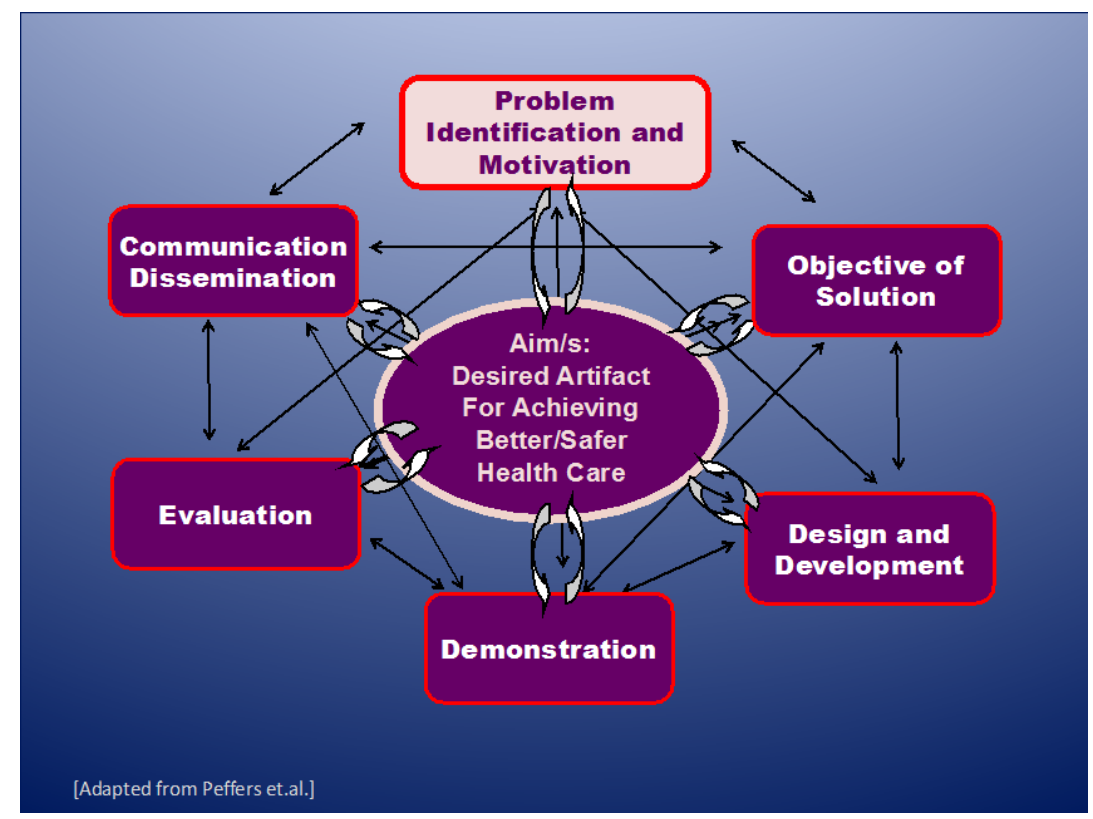

Figure 2. Portrayal of design science. 


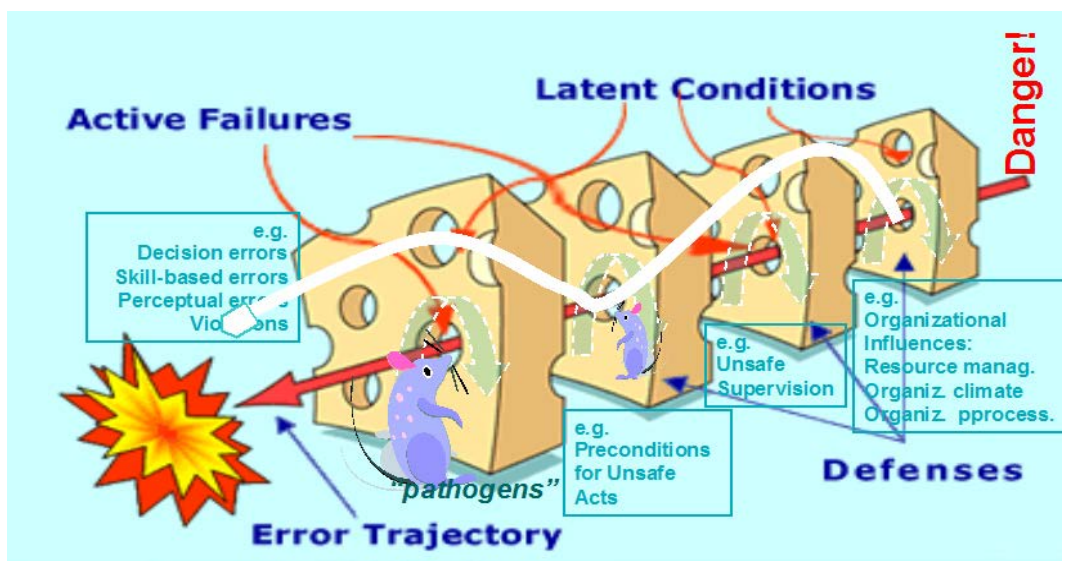

The slices of cheese represent defenses against accidents and incidents. The holes in the cheese are holes in these defenses.

Some holes are due to human errors while others

Figure 3. Reason's trajectory of errors model [20].

\section{Enhanced Perception and RESPONSE with The Safety Journal}

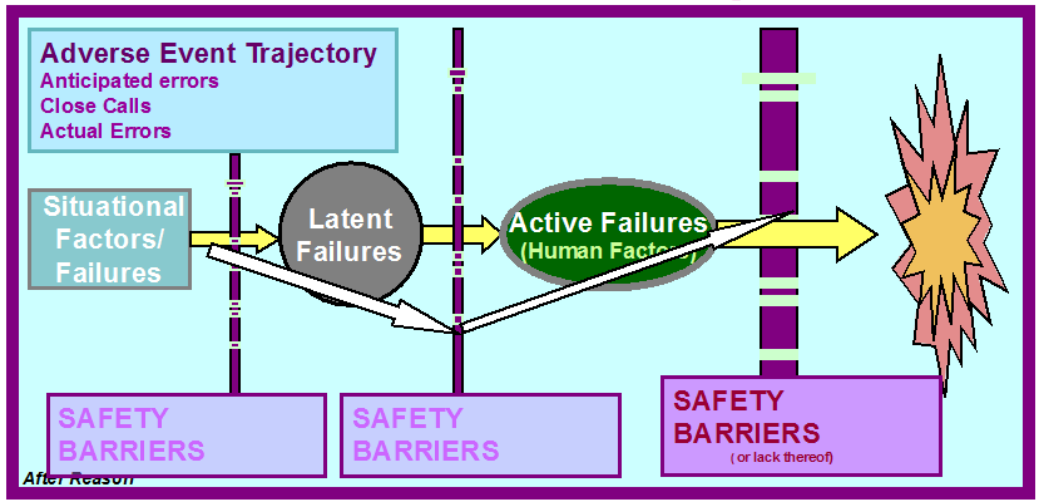

-Humans are "set up" to make errors that they are not responsible for. -Most errors result from faulty systems rather than human error.

Figure 4. Adapted trajectory of error.

and Accountability Act (HIPAA) regulations. It provides secure login, audit logs, user/reporter privacy, and role-based views of reports.

Contents of Figure 5 are incorporated in this design to reduce cognitive and emotive biases. This can be seen in the top left hand corner of Figure 6.

Figure 6 shows some of the screen shots of this interactive and user friendly prototype.

\section{Discussion}

During the 1990's researchers in organizational behavior began to understand the cultural factors at the core of the success of organizations and recognized that these factors could have applicability in whole variety of complex, risk-prone enterprises such as healthcare. The core concepts that are thought to underlie the excellence in high reliability organizations, first described by Weick and colleagues in 1999 are: 1) sensitivity to operations; 2) reluctance to simplify interpretations; 3) pre-occupation with failure; 4) deference to expertise; and 5) resilience. 
Situational Factors

These are unlucky, unusual, or unexpected events or circumstances that put additional stress on the system and can thereby trigger errors.

The additional stress placed on the system by situational factors can unmask latent failures (see below) that may have gone unnoticed for prolonged periods of time.

Examples are:

very unusual workload

distraction

unusual encounter: 2 patients with the same name arriving at the same

busy facility requiring blood

power supply failure at critical juncture

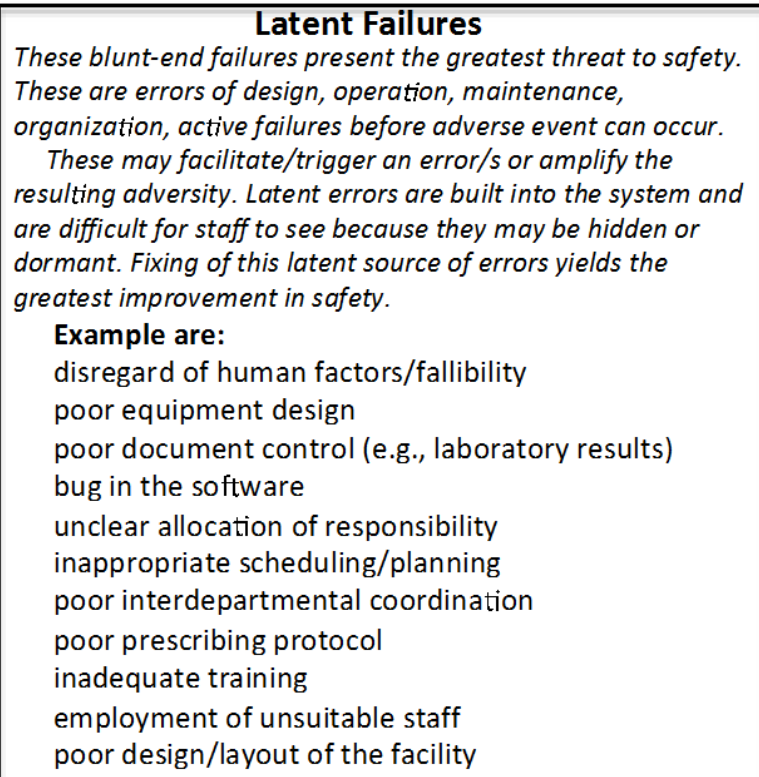

\section{Active Failures}

These sharp-end errors are made by humans/operators in

the processes of care.

Their effects are felt/observed relatively rapidly or immediately. Numerous human factors bear directly on active errors.

Examples are:

misdiagnosis

inappropriate treatment

wrong drug prescribed

wrong dose or route

wrong site procedure

picking up wrong bottle from shelf

misreading and miswriting

\section{Safety Barriers}

They hinder humans from committing an active failure or absorb the effects of active failures before adverse event

can occur. They can be: Technical (strong) or policies and procedures (week)

Technical (strong) barrier examples are:

plug design that prevents erroneous connections

equipment failing to safe default state

forcing functions

informatics

safe and distinct storage of drugs

safe dosage packaging

safety guards

intuitive equipment operation

Administrative (weak) barrier examples are:

standard protocols and procedures

clear clinical pathways

adequate credentialing requirements

emergency drills, simulations

redundancy: double checks, teach-backs

smaller number of handoffs

smaller number of links in the procedures

everything transparent and visible

adequate looseness in coupling

autonomy to handle the unexpected

nonhierarchical team culture

Figure 5. Examples of situational factors, active failures, latent failures and safety barriers.

The essential thrust of these concepts is that all workers should understand: a) the complexity of the systems within which they work and b) the proneness of such systems to fail, leading to adversity/ies. They should develop situational awareness of possibilities for incidents and should report them promptly, even if no adversity occurs. Decision-making should incorporate expertise from whoever has it, regardless of hierarchy, and staff should be prepared to handle adverse outcomes when they occur. The prototype presented here is designed to aid formation of high reliability organizations.

The pilot testers included patients, nurses, auxiliary/support staff, pharmacists and physicians. Conclusions are based on their descriptions of experiences with the new methodology as compared to various currently used methods of reporting. Testers found our method to be easy, safe, and worthwhile [12].

\section{Conclusions}

The pilot testers of this web-based tool found it to be interactive and user-friendly. These tests have been very encouraging and have demonstrated that the tool aids reporting that is meaningful and useful at the point of care. It also promotes ACGME's core competencies [22]-[24] of “system-based practice and practice-based learning and improvement". Our objective is to prepare the minds of teams, and the individuals forming them, through our methodology that invokes "improvement science" and refrains from reductionism. 

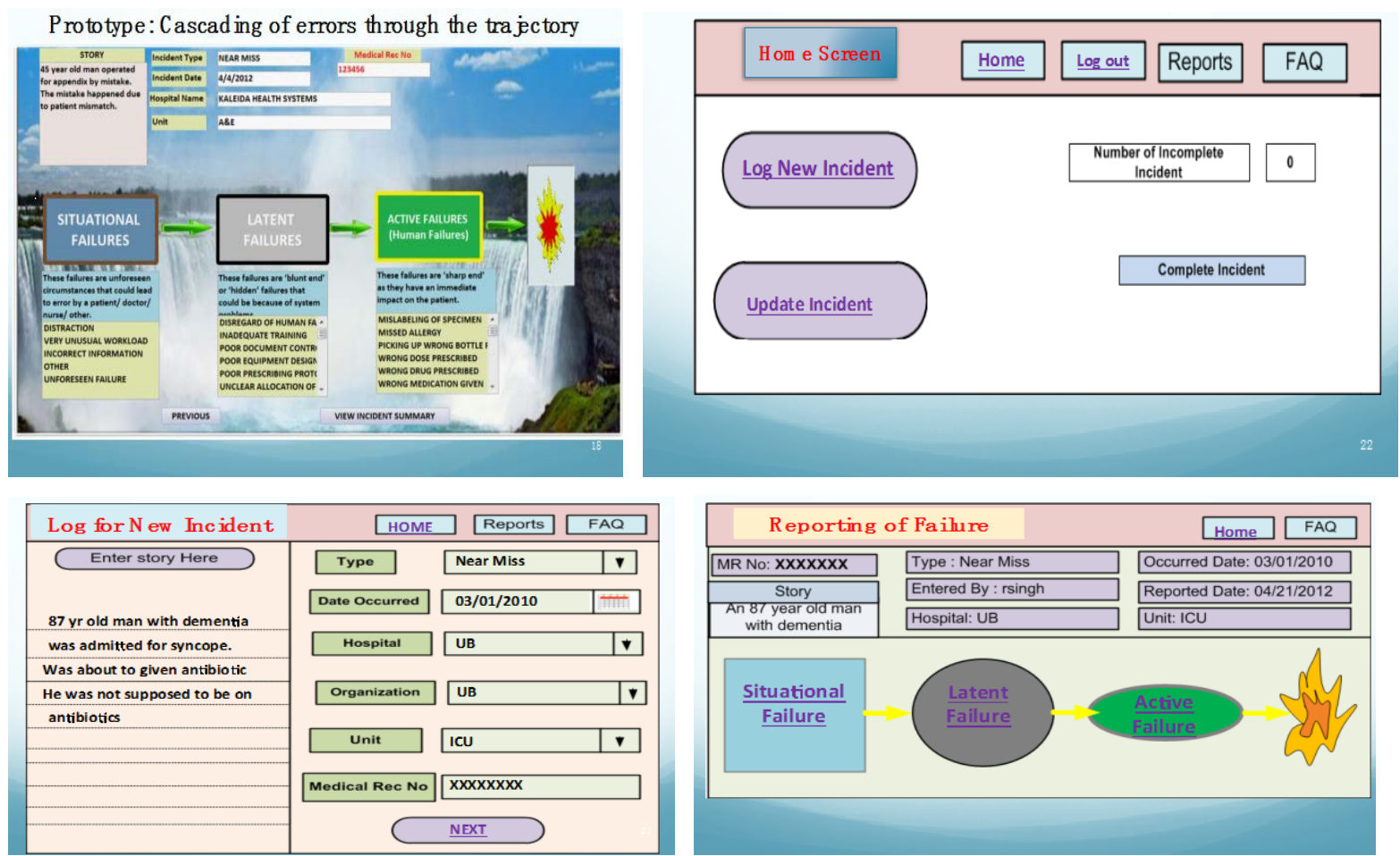

\begin{tabular}{|c|c|c|c|}
\hline \multicolumn{2}{|l|}{ Update Error } & \begin{tabular}{|l} 
Home \\
\end{tabular} & FAQ \\
\hline Story & Entered By & Date & Hospital \\
\hline 87 Year old with dementia was given & rsingh & $01 / 03 / 2010$ & UB \\
\hline 25 year old female was treated for & rsingh & 02/05/2010 & UB \\
\hline 50 Year old man with broken leg was & rsingh & $12 / 06 / 2010$ & UB \\
\hline 14 year old teenager was given wrong & rsingh & $12 / 03 / 2010$ & UB \\
\hline Select S & & Scroll Down & Scroll Up \\
\hline
\end{tabular}

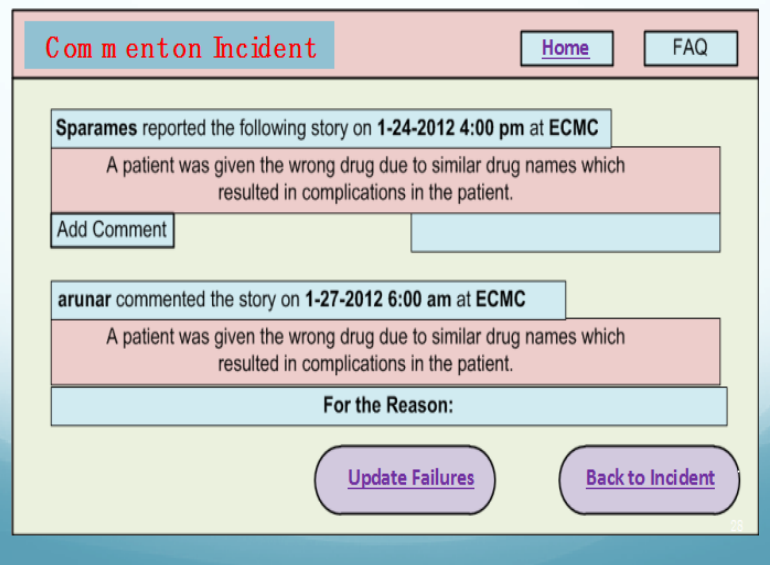

Figure 6. Some screen shots of prototype web-based reporting tool.

The author's approach is supported by and synergizes with recent recommendations by international opinion leaders [25] [26] referred to in Section 1 (paragraph 3).

Our future plans are to make the software adaptable in all healthcare settings (e.g. primary care) as well as package it for general dissemination.

\section{Acknowledgements}

The author is deeply indebted to AHRQ for funding our work. The support of his safety team is highly appreciated.

\section{References}

[1] World Health Organization (2008) World Alliance for Patient Safety. Organization Web Site.

[2] (2011) Consumer Report. New Hospital-Safety Plan Leaves Patients in the Dark.

http://news.consumerreports.org/health/2011/04/will-new-push-for-patient-safety-leave-consumers-in-the-dark.html 
[3] Office of Inspector General (2010) Adverse Events in Hospitals: National Incidence among Beneficiaries. OEI-06-0900090. http://oig.hhs.gov/oei/reports/oei-06-09-00090.pdf

[4] Editorial (2011) Medical Errors in the USA: Human or Systemic? The Lancet, 377, 1289.

[5] Kohn, L.T., Corrigan, L. and Donaldson, M.S. (2000) To Err Is Human: Building a Safer Health System. National Academies Press, Washington DC.

[6] Landrigan, C.P., Rothschild, J.M., Cronin, J.W., et al. (2004) Effect of Reducing Interns' Work Hours on Serious Medical Errors in Intensive Care Units. New England Journal of Medicine, 351, 1838-1848. http://dx.doi.org/10.1056/NEJMoa041406

[7] Balik, B. and Dopkiss, F. (2010) 10 Years after to Err Is Human: Are We Listening to Patients and Families Yet? Focus on Patient Safety Newsletter, 13, 1-3.

[8] Singh, R., Naughton, B., Singh, A., Anderson, D.R. and Singh, G. (2007) The Safety Journal: Lessons Learned with an Error Reporting Tool to Stimulate Systems Thinking. Journal of Patient Safety, 3, 135-141.

[9] Singh, R., Hickner, J., Mold, J. and Singh, G. (2014) “Chance Favors Only the Prepared Mind”: Preparing Minds to Systematically Reduce Hazards in the Testing Process in Primary Care. Journal of Patient Safety, 10, 20-28. http://dx.doi.org/10.1097/PTS.0b013e3182a5f81a

[10] Aspden, P., Corrigan, J.M., Wolcott, J., et al. (2004) Patient Safety: Achieving a New Standard for Care. National Academies Press, Washington DC.

[11] Bhasale, A. (1998) The Wrong Diagnosis: Identifying Causes of Potentially Adverse Events in General Practice Using Incident Monitoring. Family Practice, 15, 308-318.

[12] AHRQ (2011) Patient Safety Primers. The Role of the Patient in Safety. http://psnet.ahrq.gov/primer.aspx?primerID=17

[13] Clancy, C.M. (2005) Evaluating the Potential of New Technology. Building a Better Delivery System: A New Engineering/Health Care Partnership. National Academies Press, Washington DC.

[14] Snyder, L. and Neubauer, R.L., American College of Physicians Ethics, Professionalism and Human Rights Committee (2007) Pay-for-Performance Principles That Promote Patient-Centered Care: An Ethics Manifesto. Annals of Internal Medicine, 147, 792-794. http://dx.doi.org/10.7326/0003-4819-147-11-200712040-00011

[15] Kish, L. (2012) The Blockbuster Drug of the Century: An Engaged Patient. HL7 Standards. http://www.hl7standards.com/blog/2012/08/28/drug-of-the-century/

[16] Fung, C.H., Lim, Y.-W., Mattke, S., Damberg, C. and Shekelle, P.G. (2008) Systematic Review: The Evidence That Publishing Patient Care Performance Data Improves Quality of Care. Annals of Internal Medicine, 148, 111-123. http://dx.doi.org/10.7326/0003-4819-148-2-200801150-00006

[17] Hingorani, M., Wong, T. and Vafidis, G. (1999) Patients' and Doctors’ Attitudes to Amount of Information Given after Unintended Injury during Treatment: Cross Sectional, Questionnaire Survey. British Medical Journal, 318, 640-641. http://dx.doi.org/10.1136/bmj.318.7184.640

[18] US Agency for Healthcare Research \& Quality (2008) Beyond State Reporting: Medical Errors and Patient Safety Issues.

[19] Peffers, K., Tuunanen, T., Rothenberger, M.A. and Chatterjee, S. (2007) A Design Science Research Methodology for Information Systems Research. Journal of Management Information Systems, 24, 45-78.

[20] Reason, J.T. (1990) Human Error. Cambridge University Press, Cambridge. http://dx.doi.org/10.1017/CBO9781139062367

[21] Singh, R., Singh, G., McLean, D., Brennan-Taylor, M., Singh, A. and Sharman, R. (2011) Incident-Trajectory Simulation for Excellence in Safety: Illustration in Healthcare. Proceedings of the Annual Simulation Technology and Training Conference, Melbourne, 30 May-2 June 2011.

[22] Nasca, T.J., Philibert, I., Brigham, T. and Flynn, T.C. (2012) The Next GME Accreditation System—Rationale and Benefits. The New England Journal of Medicine, 366, 1051-1056.

[23] Singh, R., Naughton, B., Taylor, J.S., Koenigsberg, M.R., Anderson, D.R., McCausland, L.L., et al. (2005) A Comprehensive Collaborative Patient Safety Residency Curriculum to Address the ACGME Core Competencies. Medical Education, 39, 1195-1204. http://dx.doi.org/10.1111/j.1365-2929.2005.02333.x

[24] Singh, R., Singh, A., Fish, R., McLean, D., Anderson, D.R. and Singh, G. (2009) A Patient Safety Objective Structured Clinical Exam (OSCE). Journal of Patient Safety, 5, 55-60. http://dx.doi.org/10.1097/PTS.0b013e31819d65c2

[25] IOM (2012) Best Care at Lower Cost: The Path to Continuously Learning Health Care in America.

[26] National Advisory Group on the Safety of Patients in England (2013) A Promise to Learn—A Commitment to Act. National Health Service, UK. 